\title{
Performance of The New Integrated Front-End Electronics of the TRACE Array Commissioned with an Early Silicon Detector Prototype
}

\author{
S. Capra ${ }^{\mathrm{a}, \mathrm{b}}$, D. Mengoni ${ }^{\mathrm{c}, \mathrm{d}}$, J.A. Dueñas ${ }^{\mathrm{e}}$, P.R. John ${ }^{\mathrm{c}, \mathrm{d}, \mathrm{f}}$, A. Gadea ${ }^{\mathrm{g}}$, R.J. Aliaga ${ }^{\mathrm{g}}$, J.J. Dormard ${ }^{\mathrm{h}}$, M. Assie ${ }^{\mathrm{h}}$, A. Pullia ${ }^{\mathrm{a}, \mathrm{b}}$ \\ ${ }^{a}$ Università degli Studi di Milano, Dipartimento di Fisica, Via Celoria 16, 20133 Milano, Italy \\ ${ }^{b}$ Istituto Nazionale di Fisica Nucleare, Sez. di Milano, Via Celoria 16, 20133 Milano, Italy \\ ${ }^{c}$ Istituto Nazionale di Fisica Nucleare - Sez. di Padova, Via Marzolo 8, Padova, Italy \\ ${ }^{d}$ Dipartimento di Fisica e Astronomia, Università di Padova, Via Marzolo 8, Padova, Italy \\ ${ }^{e}$ Departmento de Ingeniería Eléctrica y Centro de Estudios Avanzados en Física, Matemáticas y Computación, Universidad de Huelva, 21071 Huelva, Spain \\ ${ }^{f}$ Institut für Kernphysik, Technische Universität Darmstadt, Darmstadt, Germany \\ ${ }^{g}$ Instituto de Física Corpuscular (CSIC-UV), C/Catedrático José Beltrán 2, Paterna, Spain \\ ${ }^{h}$ Institut de Physique Nucléaire d'Orsay, 15 rue Georges Clemenceau, Orsay, France
}

\begin{abstract}
The spectroscopic performances of the new integrated ASIC (Application-Specific Integrated Circuit) preamplifiers for highly segmented silicon detectors have been evaluated with an early silicon detector prototype of the TRacking Array for light Charged Ejectiles (TRACE). The ASICS were mounted on a custom-designed PCB (Printed Circuit Board) and the detector plugged on it. Energy resolution tests, performed on the same detector before and after irradiation, yielded a resolution of $21 \mathrm{keV}$ and $33 \mathrm{keV}$ FWHM respectively. The output signals were acquired with an array of commercial 100-MHz 14-bit digitizers. The preamplifier chip is equipped with an innovative Fast-Reset device that has two functions: it reduces dramatically the dead time of the preamplifier in case of saturation (from milliseconds to microseconds) and extends the spectroscopic dynamic range of the preamplifier by more than one order of magnitude. Other key points of the device are the low noise and the wide bandwidth.
\end{abstract}

Keywords: ASIC, Charge-Sensitive Preamplifier, Low-Noise Applications, Particle Spectrometry, Dead Time, Silicon Detector

\section{Introduction}

One of the main research topic in nuclear physics is the study ${ }^{29}$ of nuclear shell structure moving away from the valley of $\beta$ sta- ${ }^{30}$ bility. This will allow a better comprehension of nuclear reac- ${ }^{31}$ tions of astrophysical interest. In fact, even if exotic radioactive ${ }^{32}$ nuclei do not naturally occur on Earth, they are constantly gen- ${ }^{33}$ erated in stars and play a key role in the stellar reactions [1]. ${ }^{34}$ For these reasons new facilities for the production of the re- ${ }^{35}$ quired radioactive ion beams (RIBs) have been developed such ${ }^{36}$ as SPES [2] at Legnaro National Laboratories, ISOLDE [3] at ${ }^{37}$ CERN, Riken [4] (Japan), FAIR [5] (Germany) and FRIB [6] ${ }^{38}$ (MSU).

In parallel, new cutting-edge detector arrays have to be de- ${ }^{40}$ signed to comply with the new challenging measurements at 41 the RIB facilities. High-resolution $\gamma$-ray spectroscopy is one 42 of the powerful tools to study the nuclear structure. Recently, ${ }_{4}$ huge improvements in the energy resolution, peak-to-total ra- 44 tio $(\mathrm{P} / \mathrm{T})$, efficiency and ability to sustain also large counting 45 rates have been obtained thanks to the $\gamma$-ray tracking princi- 46 ple. Typically, large $\gamma$-ray spectrometers like AGATA [7] or 47 GALILEO [8] are coupled with other complementary detec- 48 tors like light-charged particle spectrometers (TRACE [9], EU- 49 CLIDES [10], DIAMANT [11]) and neutron detectors (Neu- 50 tron Wall [12] and NEDA [13]) to increase the overall resolv- 51 ing power. The detection of reaction ejectiles with highly seg- 52 mented detectors allow the precise Doppler correction of in- 53
27 flight emission of gamma-rays. Measuring simultaneously the angle and the energy of the recoil, the total kinetic energy loss can be reconstructed. This allows to obtain the energy of the state previously populated. Transfer reactions are a type of direct reactions where, by detecting the angular distribution and the energy of the particles, it is possible to infer properties of the excited states like the transferred angular momentum, and infer the spectroscopic factor. Some of the best existing examples of particle arrays for direct reactions are the MUST2 [14] and TIARA detectors [15]. When the energy resolution is insufficient to discriminate among excited states a $\gamma$-ray spectrometer is mandatory. This is the case of the heavier masses and higher level density of the nuclei produced at the new ISOL (Isotope Separation On-Line) facilities.

Highly-segmented silicon arrays are expected to be essential devices in direct reactions at RIBs facilities. Currently two new projects exist aiming at designing a state-of-the-art $4 \pi$ array in a telescope configuration: TRACE [9] and GASPARD [16]. The efforts of the two research groups are recently converging into a common project, GRIT, fully integrable with $\gamma$-ray spectrometers. GRIT will rely on the digital techniques for the particle discrimination, namely the pulse-shape analysis (PSA) approach, and consist in DSSSDs (Double-Sided Silicon Strip Detectors) having trapezoidal or square shape. The square DSSSDs are arranged in a 8-detector ring around $90^{\circ}$ while the trapezoidal DSSSDs are covering the forward and backward hemisphere. Such configuration accounts for a number of chan- 
Table 1: Summary of the specifications of the front-end electronics required by the TRACE array.

\begin{tabular}{l|c}
\hline Power consumption & $<15 \mathrm{~mW} /$ channel \\
\hline $\begin{array}{l}\text { Dimensions of one } \\
\text { FEE channel }\end{array}$ & $\begin{array}{c}<4 \times 4 \mathrm{~mm}^{2} \\
\text { (lower than detector } \\
\text { segmentation pitch) }\end{array}$ \\
\hline Equivalent noise charge & $\leq 180$ electrons rms \\
\hline Risetime (10\% - 90\%) & $<15 \mathrm{~ns}$ \\
\hline Energy dynamic range & $\begin{array}{c}\text { Linear: } \approx 40 \mathrm{MeV} \\
\text { Fast-reset: }>200-300 \mathrm{MeV}\end{array}$
\end{tabular}

\section{2.1. The technology choice}

nels larger than 10000 .

The custom ASIC (Application-Specific Integrated Circuit) multichannel preamplifier, which is described in the present ${ }_{107}$ manuscript, has been designed for an early silicon prototype of TRACE, consisting of detectors with a $20 \times 50 \mathrm{~mm}^{2}$ surface di- ${ }^{108}$ vided in 60 anodic pads and 1 common cathodic electrode. This preamplifier is foreseen to be part of the GRIT array front-end ${ }_{111}^{110}$ electronics (FEE). More specifically, it will be used to acquire the signals from the $\mathrm{E}$ layer of the telescopes.

Taking into account the number of channels, the dimensions and heat dissipation capabilities of the reaction chamber, the ${ }_{115}$ bandwidth requirements and the characteristics of the detectors, the required specifications of the front-end electronics are reported in Tab. 1. In a first place, a low-power integrated solution for the front-end electronics was adopted due to the ${ }_{119}$ restrictive requirements.

\section{Materials and methods}

In this section the integrated charge-sensitive preamplifier ${ }_{124}$ (CSP) is described, along with the custom preamplifier board ${ }_{125}$ and the TRACE detector. Moreover, a detailed description is ${ }_{126}$ given of the implemented fast-reset device that boosts the en- ${ }_{127}$ ergy dynamic range. Finally, energy reconstruction algorithm ${ }_{128}$ is explained.

This chip is designed in Austria Microsystems C35 technol-133 ogy due to two main reasons. First, this technology has $\operatorname{good}_{134}$ noise specifications at a competitive price/performance ratio. 135 Second, it provides, beside a 3.3 V-tolerant module suited for ${ }_{136}$ digital circuits, a $5 \mathrm{~V}$-tolerant module that ensures a good out- 137 put voltage swing to analog circuits. These characteristics are ${ }_{138}$ relevant since low noise and high dynamic range are two im-139 portant requirements of spectroscopy preamplifiers. The most 140 modern and scaled CMOS technologies are not always the best ${ }_{141}$ choice for the design of analog devices. The transistor scaling ${ }_{142}$ brings to digital circuits some obvious benefits like the reduction of area occupation and power dissipation. Unfortunately ${ }^{143}$ the more scaled the technology is, the lower is the maximum ${ }_{144}$ voltage tolerance of the devices. This leads to the reduction of ${ }_{145}$ the maximum power supply voltage and consequently the reduction of the output dynamic range of analog circuits. For instance, technologies with channel length of $130 \mathrm{~nm}$ hardly have supply voltages higher than $1.2 \mathrm{~V}$.

\subsection{Main features of the charge-sensitive preamplifier}

The circuit is powered with a dual $\pm 2.5 \mathrm{~V}$ voltage supply and has an area occupation of approximately $5 \mathrm{~mm}^{2}$. The power consumption is $11 \mathrm{~mW} /$ channel. The chip comprises four channels for individual pads and one for the common opposite electrode, which is separately powered and can be switched off if it is unneeded. The feedback capacitor, the bandwidth and some other key parameters of the circuit are adjustable with simple digital streams thanks to an $\mathrm{I}^{2} \mathrm{C}$ interface embedded on the chip. In this way the energy range of the CSP can be chosen among the following values: $8,20,28$ and $40 \mathrm{MeV}$. The possibility to adjust the bandwidth enables to minimize the preamplifier overshoot keeping the risetime as low as possible with different detector capacitances. In our previous work [17], experimental tests on a dedicated test-bench demonstrated that with $4 \mathrm{pF}$ of detector capacitance the output signal has a risetime $(10 \%-$ $90 \%$ ) of approximately $10 \mathrm{~ns}$. The preamplifier is equipped with a fast-reset device that boosts the spectroscopic energy range above the natural saturation threshold of the preamplifier. If a highly-energetic event saturates the preamplifier, it switches automatically to a different readout method that has proven experimentally to be linear up to $700 \mathrm{MeV}$. A precise current generator discharges the input node at constant rate. The amount of charge removed is evaluated measuring the duration of the reset procedure.

\subsection{The dedicated preamplifier board}

We mounted the ASICs on a dedicated PCB (Printed Circuit Board) [18] (see Fig. 1) that can host eight integrated preamplifiers, for a total of 32 pads plus the common electrode. The channel for cathodic signals is active only in one of those chips while the others are not powered. All the anodic channels are AC coupled thanks to decoupling capacitances directly integrated on the detector. The cathodic channel is AC coupled to the detector through a high-voltage $100 \mathrm{nF}$ X7R ceramic capacitor. The board is manifactured with a Rogers 4003C laminate. This material ensures good noise performance due to its low dielectric dispersion coefficient $\left(\epsilon_{r}=3.38\right)$ and high surface and volume resistivity $\left(4.2 \cdot 10^{9} \mathrm{M} \Omega\right.$ and $1.7 \cdot 10^{10} \mathrm{M} \Omega \mathrm{cm}$ respectively). Moreover, it is fully compatible with FR4 fabrication processes. The board is based on a 4-layer design. Great care was taken in minimizing the parasitic capacitance of connections between detector and preamplifier input. Proper shielding and per-chip active power supply filtering were added in order to avoid cross talk between different channels. The output signals are carried by three MDR (Mini Delta Ribbon) connectors. The board input connection scheme is compatible with the connector diagram of the TRACE detector prototypes.

\subsection{The TRACE detector}

The detector was produced at FBK-IRST [19] using nsubstrate float-zone technique (FZ), having a thickness of 


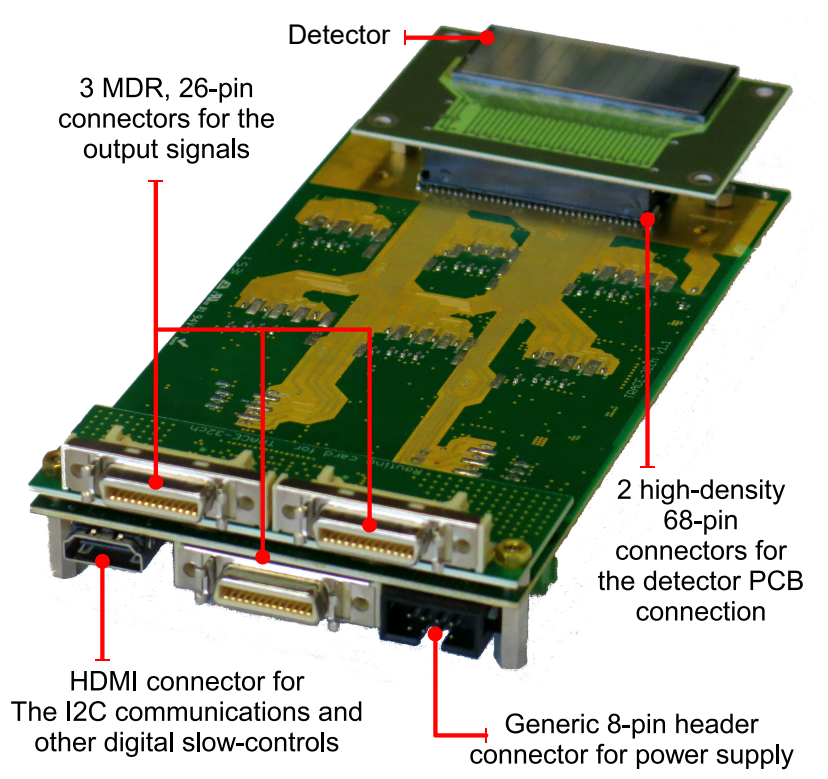

Figure 1: Picture of the TRACE32ch v1.1 preamplifier board. The different type of connectors are indicated with their own purpose. The sockets for the ${ }^{161}$ chips are located on the bottom side of the board and not visible in the figure. 162

Figure 2: Sketch of the detector's vertical view. The decoupling capacitor for the front pads is realized with a layer of aluminum deposited over an $\mathrm{SiO}_{2}$ layer ${ }^{176}$ that insulate it from the anodic implant. The thickness of the Al layer is around ${ }^{177}$ $400 \mu \mathrm{m}$ while the $\mathrm{SiO}_{2}$ layer is $700 / 800 \mu \mathrm{m}$ thick.
$200 \mu \mathrm{m}$ with a total active area of $20 \times 50 \mathrm{~mm}^{2}$. Its junction side electrode is divided in 60 square segments ( 12 by 5 pads) ${ }_{182}^{181}$ with a $4 \mathrm{~mm}$ pitch, while the ohmic side has only one electrode covering all the active area. According to manufacturer the dead layer is $<1 \mu \mathrm{m}$ and its resistivity is greater than $15^{84}$ $\mathrm{k} \Omega \cdot \mathrm{cm}$. The depletion voltage was estimated to be $15 \mathrm{~V}$, yielding a bulk capacitance of $3 \mathrm{pF}$ with a measured leakage current ${ }_{187}^{186}$ of $3 \mathrm{nA}$. More details about the TRACE detector can be found in [9]. A sketch of the detector's vertical view can be found in ${ }_{189}$ Fig. 2.

\subsection{Unit cell preamplifier}

Each channel of the ASIC preamplifier consists of threeros building blocks: an operational amplifier, a Schmitt trigger and ${ }_{194}$ a current sink (see Fig. 3). A high-bandwidth low-noise opera-195 tional amplifier is the core of the charge-sensitive preamplifier. 196

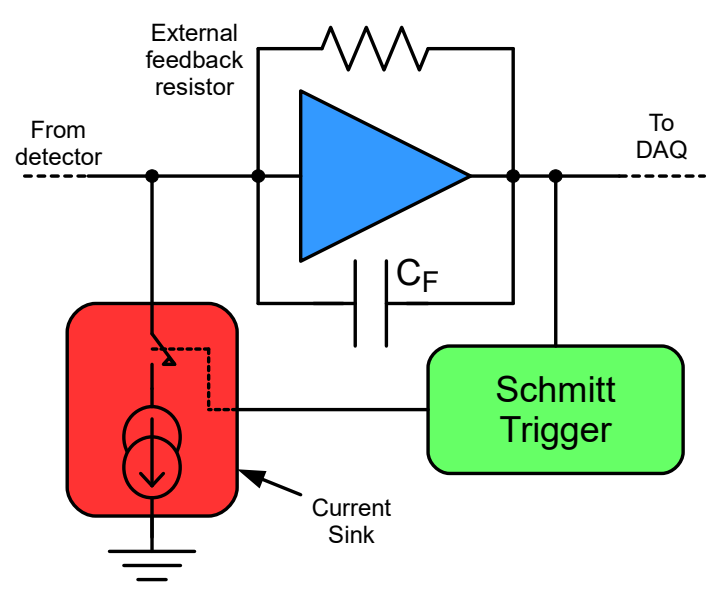

Figure 3: Block diagram of one preamplifier channel. The operational amplifier is colored in blue, the Schmitt trigger in green and the current sink in red. The operational amplifier is equipped with a low-impedance output stage able to drive a $50 \Omega$ terminated coaxial cable.

All the components are integrated except the feedback resistor that is kept as an external discrete SMT (Surface Mount Technology) device for linearity and noise reasons. If this preamplifier works in normal linear condition, the other two blocks are in an idle state and do not affect the signals. The output waveforms have an usual exponential shape that can be processed with a spectroscopy shaping amplifier, according to the classic analog processing techniques, or directly acquired with an ADC (Analog-to-Digital Converter) in full-digital acquisition systems.

\subsection{Fast-reset procedure}

The issues related to signal saturation are more frequent and problematic in integrated devices respect to discrete ones because of their limited power supply voltages and, consequently, their limited output dynamics. In this chip, the role of the comparator is to sense when the operational amplifier is running in saturation. A pre-defined threshold delimits the boundary of the linear operation region. When the comparator recognizes that the CSP output signal has crossed the saturation threshold, it activates the current sink, letting the reset process begin. The current sink is a precise constant current generator. Its working principle is quite straightforward: an operational amplifier keeps the voltage across a reference resistor constant. The current generated by the resistor is collected by a MOS (metaloxide semiconductor) transistor and used to perform the reset.

When the current sink is activated, it starts to drain out charge from the input node. According to the amount of charge released by the detector, the preamplifier remains saturated for a variable amount of time. Once all the excess charge has been removed from the input node, the output stage of the operational amplifier comes back in operating condition, producing a ramp-like signal: it represents the charge being removed at constant rate from the input node. The reset process ends when the output signal crosses a second pre-defined threshold: the comparator switches and the current sink is disconnected from 


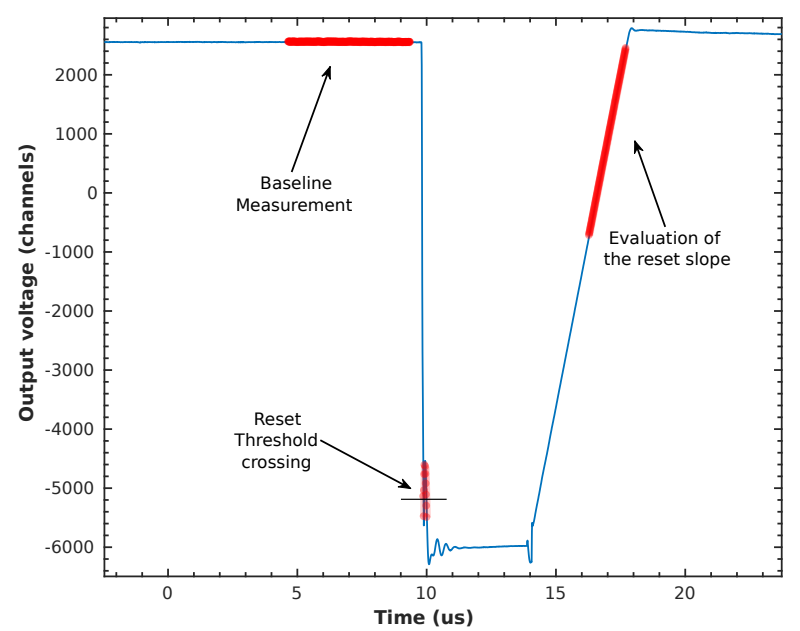

Figure 4: Reset procedure of the integrated charge sensitive preamplifier. A pulser is connected through a test capacitor of $1 \mathrm{pF}$ to the input node of the CSP. The pulser simulates events with equivalent energy released in silicon equal to $110.8 \mathrm{MeV}$. The reset procedure lasts $7.8 \mu \mathrm{s}$. the input node. From this moment on the preamplifier is imme- ${ }^{230}$ diately free to work in its usual operating mode. A complete ${ }^{231}$ reset cycle of the integrated preamplifier is represented in Fig. ${ }^{232}$ 4. The whole reset procedure generally takes some microsec- ${ }^{233}$ onds, according to the event energy. The reset speed can be $\mathrm{e}^{234}$ adjusted through $\mathrm{I}^{2} \mathrm{C}$ control in a range between $1.5 \mathrm{MeV}_{\mu \mathrm{s}}{ }^{-1235}$

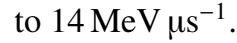

Generally the dead-time of a preamplifier can last some mil- ${ }^{23}$ liseconds according to the CSP's dynamic range and charge re- ${ }^{238}$ leased by the detector. The fast-reset procedure reduces this ${ }^{23}$ time roughly by a factor between $10^{2}$ and $10^{3}$. Reduction of ${ }^{240}$ dead-time is not the only benefit of fast-reset circuit. This one $e^{241}$ can also be employed to extend the dynamic range of the pream- ${ }^{242}$ plifier. When an event inside the detector releases a relevant amount of charge, causing the preamplifier saturation, the output signal is distorted. The energy information cannot be retrieved from the output signal. However, the information is still ${ }^{243}$ contained in the amount of charge released by the detector and $\mathrm{d}^{244}$ trapped on the input node of the preamplifier. This charge is ${ }^{245}$ not lost: can be collected and measured. If the current genera $-{ }^{246}$ tor used to perform the reset procedure is precise and constant, ${ }^{247}$ it is possible to measure the amount of charge removed from ${ }^{248}$ the input node measuring the reset process duration.

\subsection{Energy reconstruction algorithm}

An innovative algorithm to retrieve the energy information ${ }_{253}$ even in case of deep saturation has been developed. It recon- ${ }_{254}$ structs the triangular shape of the signal during the reset process 255 like if the preamplifier had an unlimited voltage swing. For a pictorial view of the algorithm see Fig. 5. The energy of the event is proportional to the amplitude of the voltage step $(\Delta \mathrm{V})$ that would occur if the preamplifier was linear and not satu-256 rated.

$$
E_{(\text {event })}=K \cdot C_{F} \cdot \Delta V
$$

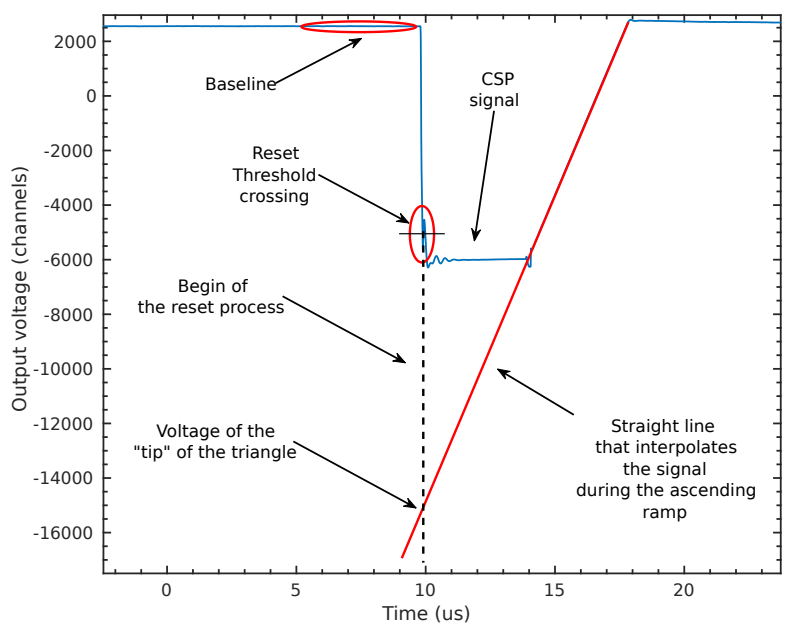

Figure 5: Pictorial view of the algorithm used to reconstruct the energy of fastreset signals.

$\Delta \mathrm{V}$ is the difference between the baseline voltage $\left(V_{B L}\right)$ and the "tip" $\left(V_{T I P}\right)$ of the triangular waveform ideally produced during the fast-reset signal if the preamplifier had infinite dynamic range. $\mathrm{K}$ is the proportionality factor between charge released by the detector and the corresponding energy. $C_{F}$ is the value of feedback capacitance.

In order to obtain $\mathrm{V}_{T I P}$, the parameters of a straight line that best fits the ascending ramp is calculated. Such straight line is then evaluated in $t_{S T A R T}$, which is the instant when the real signal crosses the reset threshold and activates the current generator. The parameter $t_{S T A R T}$ is obtained interpolating two or more points of the signal. The reset threshold voltage is a wellknown measurable quantity. The time-dependent function $y_{S L}$ that best fits the ascending ramp is a straight line:

$$
y_{S L}(t)=S_{R A M P} \cdot t+q .
$$

The slope of the ascending ramp $\mathrm{S}_{R A M P}$ is constant for each preamplifier channel and calculated on a large number of experimental signals. The $q$ parameter depends on the reset duration and, consequently, on the event energy. A proper power supply filtering and regulation have demonstrated experimentally to make the reset device robust enough to be used in a 24-hour acquisition without appreciable drift of the $S_{R A M P}$ parameter. It is not advisable to operate these chips with unregulated power supplies because this would degrade significantly the resolution in fast-reset mode. For each signal, the best value of $q$ is calculated on a collection points from the ramp called here as " $G$ ". The number of points chosen can vary according to the required accuracy and available computational power.

$$
\langle q\rangle=\left\langle y(i)-S_{R A M P} \cdot t(i)\right\rangle
$$

In equation $4 y(i)$ is the voltage and $t(i)$ is the timestamp of the i-th point in the collection G. The best value for the voltage at the tip of the triangle is: 


$$
\begin{aligned}
V_{T I P} & =\left\langle y_{S L}\left(t_{S T A R T}\right)\right\rangle= \\
& =\sum_{i \in G} \frac{\left[y(i)-S_{R A M P} \cdot\left(t(i)-t_{S T A R T}\right)\right]}{n} .
\end{aligned}
$$

After this procedure we end up with two spectra. The first is produced applying moving-window deconvolution algorithms on the exponential signals, the second is calculated processing over-threshold fast-reset events. Combining these two, a unique calibrated spectrum is obtained. In this way, even if the natural dynamic range of the preamplifier is $40 \mathrm{MeV}$, the portion of the spectra calculated with fast-reset events can be extended much further, up to several hundreds of MeV. Since the duration of the fast-reset events is energy-dependent, the spectroscopic energy limit of this procedure is determined by three factors: reset speed, length of the acquisition window and ESD (Electro-Static Devices) protection structures on the input node of the preamplifier. The algorithm to reconstruct the energy of fast-reset events is essentially based on a time measurement: the higher the reset speed, the lower the accuracy. In the same way, the acquisition window lenght may determine the upper measurable energy for a given reset speed. The reset current308 choice is thus a compromise between energy range and resolu-309 tion. The only physical energy limit of the fast-reset device is 310 the charge loss caused by the ESD structures. When the CSP ${ }_{311}$ saturates and the feedback capacitor is not able to collect all the 312 charge released by the detector, this charge remains trapped on 313 the input node, causing a voltage bounce. If this voltage bounces 14 is high enough to activate the ESD structures of the input tran-315 sistor, some of the charge is unavoidably lost through protection 316 diodes.

The results reported in the next section demonstrate that ${ }_{318}$ this technique can be used in combination with a preampli- ${ }_{319}$ fier equipped with the fast-reset device in actual experimental ${ }_{320}$ conditions in order to produce spectral lines with FWHM (Full ${ }_{321}$ Width at Half Maximum) equal to $0.2 \%$ of the energy or lower. ${ }_{322}$

\section{Experimental results}

In this section the experimental results are presented. They ${ }_{326}$ cover three topics: the spectrum acquisition of an $\alpha$ source,327 the evaluation of the preamplifier equivalent noise charge with-328 out detector and the acquisition of a wide-energy-range, pulser- 329 produced spectrum. The measurements in this work are related 330 to the anodic channels only: the cathodic channel was used just 331 for triggering purposes.

\section{1. $\alpha$ source spectrum acquisition}

In order to evaluate the spectroscopic performance of the in-33 tegrated charge-sensitive preamplifiers, the spectrum of a mixed ${ }^{336}$ nuclide $\left({ }^{239} \mathrm{Pu},{ }^{241} \mathrm{Am},{ }^{244} \mathrm{Cm}\right)$ alpha source placed in front of ${ }^{337}$ the junction side was acquired with a TRACE detector pro-3з8 totype connected to the TRACE32ch v1.1 preamplifier board. 339 The detector was plugged directly on the board and operated in $_{340}$ a vacuum chamber together with the alpha source. The detector 341 bias voltage was produced using a CAEN N1470 module that 342

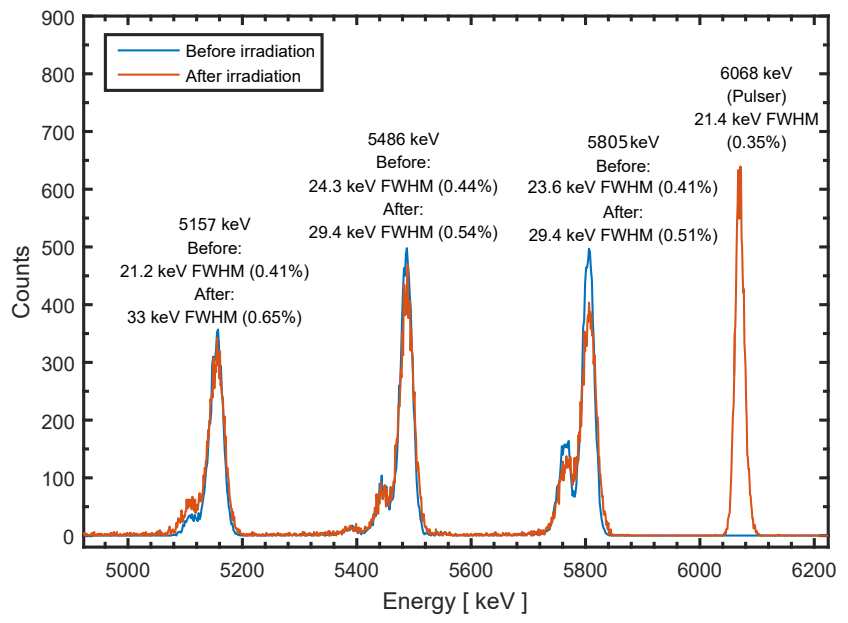

Figure 6: Spectrum of a mixed nuclide $\left({ }^{239} \mathrm{Pu},{ }^{241} \mathrm{Am},{ }^{244} \mathrm{Cm}\right)$ alpha source. Detector biased with $40 \mathrm{~V}$ never irradiated before. The calibration was performed with a linear fit on the centroids of the three main peaks and the pulser line.

allows to monitor the current with an accuracy of $2 \mathrm{nA}$. The detector bias was fixed at $40 \mathrm{~V}$. Custom MDR to $3 \mathrm{M}$ header adapters were used to connect the board to the connectors on the chamber's flange. An array of CAEN N1728A digitizer cards was used to acquire and store the signals. These are FPGApowered, $100 \mathrm{MHz}$ 14-bit, 4-channel digitizer cards with differential inputs. The output signals from the preamplifier board were sampled and processed with a trapezoidal filter that has a $3 \mu$ s-wide flat top and $8 \mu$ s risetime.

After the first acquisition the detector was used in an in-beam experiment [20]. The irradiation details are summarized in Tab. 3 . The leakage current of the detector before irradiation was so low to be barely measurable and can be considered to be equal to $1 \mathrm{nA}$. After irradiation, the leakage current was $0.618 \mu \mathrm{A}$. The spectra acquired before and after the in-beam experiment are reported in Fig. 6. The corresponding energy resolutions are reported in Tab. 2. In the setup with the irradiated detector, a pulser line was added. A pulse signal with peak-to-peak amplitude of $230 \mathrm{mV}$ was injected through a $1 \mathrm{pF}$ test capacitor on the input node of the preamplifier. The calibration of the spectrum $\mathrm{x}$-axis was obtained using a simple linear fit algorithm on the three main peaks centroids. Considering that the measurements (before and after irradiation) were performed using different digitizer boards with two intrinsically different analog gains, in Fig. 6 the calibration is different for the two spectra. A spectrum calibration on the three main peaks of the alpha source enables us to evaluate the equivalent energy of the pulser peak $(6068 \pm 20 \mathrm{keV})$ with a relative error of $\pm 0.3 \%$. The ratio between pulser voltage and equivalent energy in silicon is used also to calibrate the spectrum in Fig. 9.

The effect of radiation damages consists in the detector resolution rising from roughly 24 to $29 \mathrm{keV} \mathrm{FWHM}$ at $5.5 \mathrm{MeV}$. Looking at the results in Fig. 6, one must take into account that the FWHM energy straggling induced by a $0.4 \mu \mathrm{m}$ Aluminum layer on $5.5 \mathrm{MeV}$ alpha particles is equal to $14.18 \mathrm{keV}$. The 
Table 2: Peak Energies and resolutions of the ${ }^{241} \mathrm{Am}-{ }^{244} \mathrm{Cm}-{ }^{239} \mathrm{Pu}$ alpha spectrum of Fig. 6 before and after detector irradiation. Only the three major peaks were considered.

\begin{tabular}{ccc}
\hline $\begin{array}{c}\text { Alpha particle } \\
\text { energy } \\
{[\mathrm{keV}]}\end{array}$ & $\begin{array}{c}\text { Resolution } \\
\text { FWHM } \\
{[\mathrm{keV}]}\end{array}$ & $\begin{array}{c}\text { Resolution } \\
\text { FWHM } \\
{[\%]}\end{array}$ \\
\hline \multicolumn{3}{c}{ Before irradiation (Fig. } \\
\hline 5157 & 21.2 & blue line) \\
5486 & 24.3 & 0.41 \\
5805 & 23.6 & 0.44 \\
\hline \multicolumn{2}{c}{ After irradiation (Fig. 6 orange line) } \\
\hline 5157 & 33.0 & 0.65 \\
5486 & 29.4 & 0.54 \\
5805 & 29.4 & 0.51 \\
$6068 \pm 20$ & 21.4 & 0.35 \\
(pulser) & & \\
\hline
\end{tabular}

Table 3: Key parameters of the experiment that involved the TRACE detectors.

\begin{tabular}{c|c}
\hline Beam current & $1 \mathrm{pnA}$ \\
Beam Type & ${ }^{37} \mathrm{Cl}$ \\
Beam energy & $186 \mathrm{MeV}$ \\
Detector distance from target & $63 \mathrm{~mm}$ \\
Detector angle (respect to beam-line) & $35^{\circ}-57^{\circ}$ \\
Target & ${ }^{12} \mathrm{C}, 0.1 \mathrm{mg} \mathrm{cm}^{-2}$ \\
Detector average counting rate & $10 \mathrm{kHz}$ \\
Duration & $60 \pm 3 \mathrm{~h}$ \\
\hline
\end{tabular}
quadratic difference between the FWHM of the alpha lines and the one of the pulser line gives information about the resolution loss due to fundamental physical phenomena (interaction of the particles with the detector, charge collection mechanisms etc.). A quadratic difference of $20 \mathrm{keV}$ corresponds to the alpha straggling caused by a $0.8 \mu \mathrm{m}$-thick aluminum layer or by a $0.4 \mu \mathrm{m}$-thick aluminum layer followed by a $0.8 \mu \mathrm{m}$-thick $\mathrm{SiO}_{2}{ }^{365}$ layer. The latter is a reasonable option according to the detector ${ }^{366}$ specifications (see Fig. 2). The pulser line FWHM in Fig. $6_{367}$ should be determined only by the detector shot noise and the ${ }_{368}$ preamplifier's input noise. Unfortunately, this FWHM value is 369 quite high $(21.4 \mathrm{keV})$ and cannot be justified only considering ${ }_{370}$ the aforementioned noise sources. Future research is needed ${ }_{371}$ to determine the exact noise sources and their relative contri-372 butions in case both of a brand-new and an irradiated detec-з73 tor. During the acquisition with the irradiated detector, baseline ${ }_{374}$ fluctuations in the order of $100 \mathrm{mV}$ on the CSPs' signals have ${ }_{375}$ been observed with characteristic times in the order of $1 \mathrm{~ms}$ or $_{376}$ lower. Such fluctuations can be related to the detector power 377 supply (which, anyway, was highly filtered inside the vacuum 378 chamber) or to an anomalous behavior of the detector. The lat-379 ter hypothesis is likely, especially after radiation damage. $\quad 380$

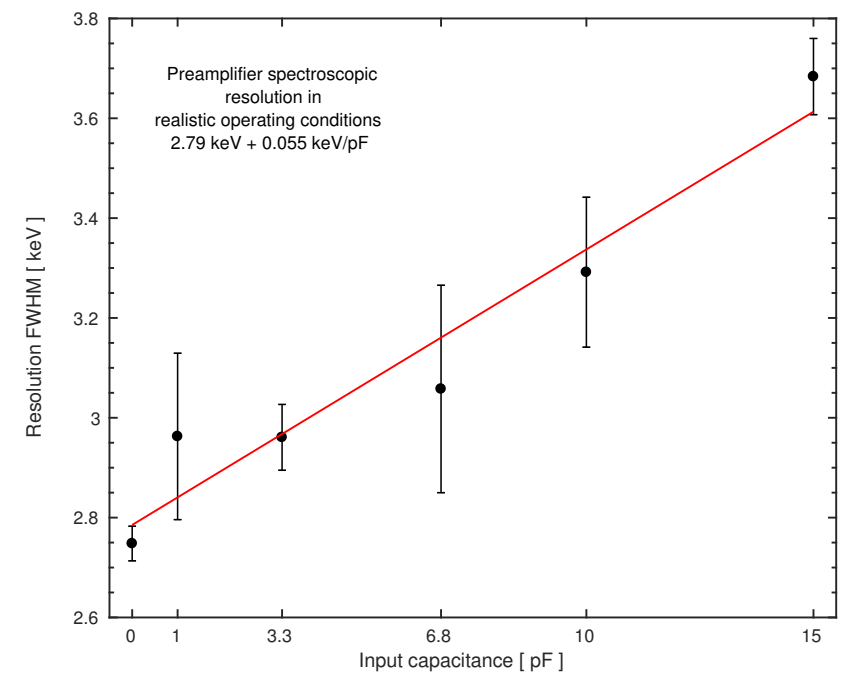

Figure 7: Resolution of the preamplifier against different input capacitances. The signal was injected in the input node with a pulser through a test capacitor of $1 \mathrm{pF}$. The pulser peaks were fitted with Gaussian functions. The error bars represent the uncertainty on the peak widths.

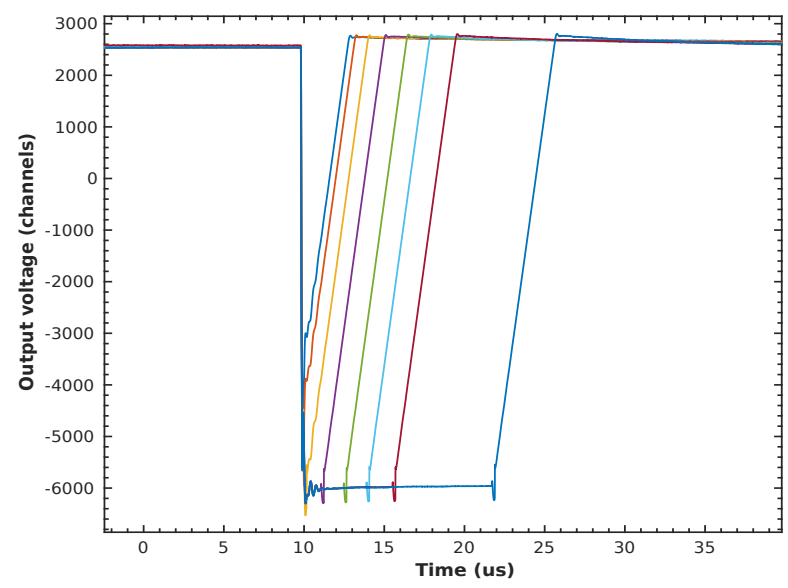

Figure 8: Output signals from the preamplifiers during the fast-reset procedure. Different reset durations are due to different event energies. Since no source was available with energy higher than $5805 \mathrm{keV}$, a pulser was used.

\subsection{Estimation of the preamplifier noise contribution without detector}

In order to estimate the noise contributions involved in the final result reported in Tab. 2, we first measured the resolution of pulser lines. The detector was unplugged from the board and precision capacitors were connected to the input node of the preamplifier. The chosen capacitances are $1 \mathrm{pF}, 3.3 \mathrm{pF}$, $6.8 \mathrm{pF}, 10 \mathrm{pF}$ and $15 \mathrm{pF}$. The results are reported in Fig. 7. The preamplifier resolution was evaluated in each case acquiring a pulser-produced spectrum with two lines at known energy (3000 events in total). The data shown in Fig. 7 were obtained with Gaussian fits on such spectra. The error bars represent the uncertainty on the peak widths given by the fitting algorithm.

In this experimental setup, the preamplifier shows a resolution of $2.79 \mathrm{keV}$ plus $0.055 \mathrm{keV}$ FWHM for every $\mathrm{pF}$ of capacitance added to the input. Considering that the estimated 


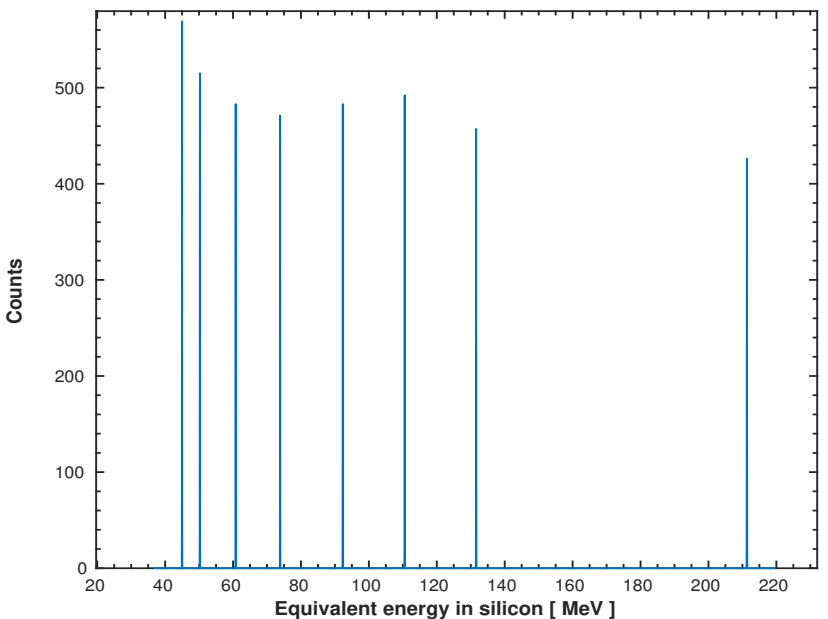

Figure 9: Spectrum of fast-reset events acquired with pulser as source. The algorithm used to retrieve the energy measurement is the one described in the ${ }^{394}$ previous section.

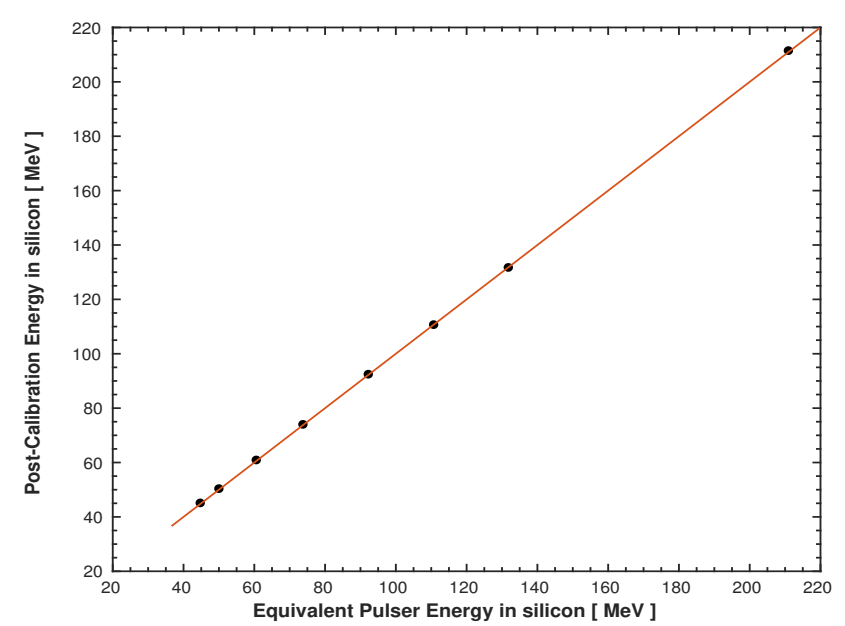

Figure 10: Linear fit of the peaks in figure 10 against the equivalent energy cal- 414 culated from the amplitude of pulser signal. The error bars are smaller than data ${ }_{415}$ points (see Tab. 4). The $\mathrm{R}^{2}$-coefficient is 0.99999 . Considering a reasonable error bar of $0.2 \%$ of the total energy, the $\chi^{2}$ test with 6 degrees of freedom gives ${ }^{416}$ a result of 6.215, equivalent to a reduced $\chi^{2}$ of 1.04 .

detector capacitance in typical operating conditions is $4 \mathrm{pF}$, we can conclude that the preamplifier contribution to the spectral ${ }^{422}$ lines FWHM is approximately $3 \mathrm{keV}$. Fig. 7 shows that the ex- ${ }^{223}$ perimental setup was non-optimal, since our previous measure- ${ }^{224}$ ments [17] demonstrated that the equivalent preamplifier resolution in silicon with $4 \mathrm{pF}$ of detector capacitance is $1.11 \mathrm{keV}^{426}$ Such result was obtained with full analog spectroscopic chain, including a semi-Gaussian shaping amplifier with $10 \mu$ shaping $_{427}$ time. This means that the noise related to the digitizer cards and the pickup noise due to the chamber-rack connections is higher ${ }_{428}$ than the one of the preamplifiers themselves. Another possible ${ }_{42}$ source of noise can be related to ground reference fluctuations 430 between the vacuum chamber and the instrumentation rack. $\quad{ }_{431}$
Table 4: Energies, residuals and resolutions of the peaks in Fig. 9. The uncertainty in the pulser energy is due to the uncertainties in the spectrum calibration of Fig. 6.

\begin{tabular}{rrrrrr}
\hline $\begin{array}{c}\text { Pulser } \\
\text { Energy } \\
{[\mathrm{keV}]} \\
\pm 0.3 \%\end{array}$ & $\begin{array}{c}\text { Best fit } \\
\text { energy } \\
{[\mathrm{keV}]} \\
\pm 10 \mathrm{keV}\end{array}$ & $\begin{array}{c}\text { Residual } \\
{[\mathrm{keV}]} \\
\pm 1 \mathrm{keV}\end{array}$ & $\begin{array}{c}\text { Residual } \\
{[\%]}\end{array}$ & $\begin{array}{c}\text { Resol. } \\
\text { FWHM } \\
{[\mathrm{keV}]} \\
\pm 1 \mathrm{keV}\end{array}$ & $\begin{array}{c}\text { Resol. } \\
\text { FWHM } \\
{[\%]}\end{array}$ \\
\hline 44850 & 44932 & -82 & $-0,18$ & 47 & 0,10 \\
50127 & 50246 & -119 & $-0,24$ & 51 & 0,10 \\
60680 & 60792 & -112 & $-0,18$ & 51 & 0,08 \\
73871 & 73886 & -14 & $-0,02$ & 52 & 0,07 \\
92339 & 92332 & 8 & 0,01 & 61 & 0,07 \\
110807 & 110559 & 248 & 0,22 & 60 & 0,05 \\
131913 & 131589 & 324 & 0,25 & 67 & 0,05 \\
211061 & 211312 & -251 & $-0,12$ & 72 & 0,03 \\
\hline
\end{tabular}

\subsection{Acquisition of a Wide-energy-range, pulser-produced spectrum}

After the measurements with the alpha source, an artificial spectrum was produced connecting a pulser to the preamplifiers input. The energies were chosen to go over threshold and activate the fast-reset device. In Fig. 8 some preamplifier output signals are shown for different equivalent pulser energies. In Fig. 9 the acquired spectrum is presented. The algorithm used to retrieve the energy of the fast-reset events is the one described in section 2. As can be seen in Tab. 4, the overall energy resolution is under $0.1 \%$ FWHM along all the spectrum which is exceptionally good respect to the requirements of TRACE. The linear fit in Fig. 10 shows that the residuals (the difference between the calibrated peak energy and the value of the fitting line at the same pulser amplitude) is negligible all across the spectrum, being in the order of $0.2 \%$ of the total energy for every peak. This result is really encouraging, especially if compared to other works, such as [21], focused on the energy reconstruction over the saturation threshold. Not only does the technological advance consist in the reduction of the relative resolution, but also in the fact that the described method involves just the analysis of the digitized preamplifier waveforms and no auxiliary signal is needed. The simultaneous digitalization of the preamplifier and comparator signals would double the required ADC channels. This solution is clearly unfeasible in modern highly-segmented detector arrays.

With $4 \mathrm{pF}$ detector capacitance and pulser risetime $<10 \mathrm{~ns}$, the upper limit of the experimental dynamic energy range is above $700 \mathrm{MeV}$.

A brief summary of all the mentioned characteristics of the ASIC preamplifier is reported in Tab. 5. The device is compliant with the requirements of the TRACE array reported in Tab. 1.

\section{Conclusions}

The circuit structure, the working principles and the experimental performance of an integrated charge-sensitive preamplifier for solid-state detectors have been presented. The resolution of the preamplifier $(1.1 \mathrm{keV}$ on dedicated test-bench, $3 \mathrm{keV}$ 
Table 5: Summary of the ASIC parameters.

\begin{tabular}{l|c}
\hline Process & $\begin{array}{c}\text { AMS } 0.35 \mu m \\
\text { 3.3/5V Mixed Signal }\end{array}$ \\
\hline Die size & $3.3 \times 1.5 \mathrm{~mm}^{2}$ \\
\hline Power supply & $\pm 2.5 \mathrm{~V}$ \\
\hline Power consumption & $11 \mathrm{~mW} /$ channel $^{-}$ \\
\hline Input stage structure & Differential \\
\hline $\begin{array}{l}\text { Number of channels } \\
\text { for anodic signals }\end{array}$ & 4 \\
\hline $\begin{array}{l}\text { Number of channels } \\
\text { for cathodic signals }\end{array}$ & $130 \mathrm{e}^{-} \mathrm{rms}$ \\
\hline $\begin{array}{l}\text { ENC } \\
\text { (anodic channels) }\end{array}$ & $(4 \mathrm{pF} \mathrm{detector} \mathrm{cap)}$ \\
\hline $\begin{array}{l}\text { ENC } \\
\text { (cathodic cannels) }\end{array}$ & $\begin{array}{c}143 \mathrm{e} \mathrm{ems}^{-} \mathrm{rms} \\
(4 \mathrm{pF} \mathrm{detector} \mathrm{cap})\end{array}$ \\
\hline $\begin{array}{l}\text { Energy resolution } \\
\text { (anodic channels) }\end{array}$ & $1.11 \mathrm{keV} \mathrm{FWHM}$ \\
\hline $\begin{array}{l}\text { Energy resolution } \\
\text { (cathodic channels) }\end{array}$ & $1.2 \mathrm{keV} \mathrm{FWHM}$ \\
\hline Selectable gains & $(4 \mathrm{pF})$ \\
\hline Linear dynamic range & $8 / 20 / 28 / 40 \mathrm{MeV}$ \\
\hline Fast-reset dynamic range & $>=700 \mathrm{MeV}$ \\
\hline Risetime (10\% - 90\%) & $9 \mathrm{~ns} \mathrm{minimum}(4 \mathrm{pF})$ \\
\hline
\end{tabular}

in non-optimal experimental setup) is very good compared to ${ }^{495}$ works from literature. Recently developed integrated pream- ${ }_{497}^{496}$ plifiers for silicon detectors have an equivalent noise charge ${ }_{498}$ (ENC) around 200 electrons rms [22] or 188 electrons rms [23]499 (respectively equivalent to $1.7 \mathrm{keV}$ and $1.6 \mathrm{keV}$ FWHM) for ${ }^{500}$ $4 \mathrm{pF}$ detector capacitance.

Both the fast-reset auxiliary device and the algorithm used to503 retrieve the energies of fast-reset events demonstrate to work as ${ }^{504}$ expected. Despite the intrinsic preamplifier saturation energy ${ }_{506}^{505}$ being $40 \mathrm{MeV}$, the fast-reset device allows for high-resolution ${ }_{507}^{506}$ spectroscopy over the saturation limit up to $700 \mathrm{MeV}$, with res-508 olution of over-threshold peaks better than $0.1 \%$ FWHM. Fu-509 ture R\&D must be focused on the design of a preamplifier ${ }_{511}^{510}$ PCB for the upcoming version of the integrated charge sensi- ${ }_{512}$ tive preamplifier. This will allow to read all the 60 detector 513 channels. The results obtained also pave the way for a future ${ }^{514}$ in-beam experiment, even with the current preamplifier, which ${ }_{516}^{515}$ can allow for an evaluation of the setup particle-discrimination ${ }_{517}$ capabilities.

\section{References}

[1] Y. Penionzhkevich, Exotic nuclei and astrophysics, Vol. 337, 2012. ${ }_{524}^{52}$ doi:10.1088/1742-6596/337/1/012046.

[2] M. Maggiore, D. Campo, P. Antonini, A. Lombardi, M. Manzolaro, ${ }^{525}$ A. Andrighetto, A. Monetti, D. Scarpa, J. Esposito, L. Silvestrin, SPES: ${ }_{527}$ A new cyclotron-based facility for research and applications with high- intensity beams, Modern Physics Letters A 32 (17) (2017) 1740010. doi:10.1142/s0217732317400107.

[3] E. Kugler, Hyperfine Interactions 129 (1/4) (2000) 23-42. doi:10.1023/a:1012603025802.

[4] T. Kubo, M. Ishihara, N. Inabe, H. Kumagai, I. Tanihata, K. Yoshida, T. Nakamura, H. Okuno, S. Shimoura, K. Asahi, The RIKEN radioactive beam facility, Nuclear Instruments and Methods in Physics Research Section B: Beam Interactions with Materials and Atoms 70 (1-4) (1992) 309-319. doi:10.1016/0168-583x(92)95947-p.

[5] H. Emling, The rare-isotope-beam facility at FAIR, The European Physical Journal Special Topics 150 (1) (2007) 235-240. doi:10.1140/epjst/e2007-00312-7.

[6] G. Bollen, Y. E. Penionzhkevich, S. M. Lukyanov, FRIB-facility for rare isotope beams, AIP, 2010. doi:10.1063/1.3431449.

[7] J. Simpson, The AGATA spectrometer: next generation gamma-ray spectroscopy, Journal of Physics: Conference Series 606 (2015) 012017. doi:10.1088/1742-6596/606/1/012017.

[8] C. A. Ur, Perspectives for the gamma-ray spectroscopy at LNL: the GALILEO project, Journal of Physics: Conference Series 366 (2012) 012044. doi:10.1088/1742-6596/366/1/012044.

[9] D. Mengoni, J. Dueñas, M. Assié, C. Boiano, P. John, R. Aliaga, D. Beaumel, S. Capra, A. Gadea, V. Gonzáles, A. Gottardo, L. Grassi, V. Herrero-Bosch, T. Houdy, I. Martel, V. Parkar, R. Perez-Vidal, A. Pullia, E. Sanchis, A. Triossi, J. V. Dobón, Digital pulse-shape analysis with a TRACE early silicon prototype, Nuclear Instruments and Methods in Physics Research Section A: Accelerators, Spectrometers, Detectors and Associated Equipment 764 (2014) 241-246. doi:10.1016/j.nima.2014.07.054.

[10] D. Testov, et al., EPJ A, Accepted for publication.

[11] J. N. Scheurer, et al., Improvements in the in-beam $\gamma$-ray spectroscopy provided by an ancillary detector coupled to a ge $\gamma$-spectrometer: The diamant-eurogam ii example, Nuclear Instruments and Methods in Physics Research, Section A: Accelerators, Spectrometers, Detectors and Associated Equipment 385 (3) (1997) 501-510. doi:10.1016/S01689002(96)01038-8.

[12] Ö. Skeppstedt, et al., The euroball neutron wall - design and performance tests of neutron detectors, Nuclear Instruments and Methods in Physics Research, Section A: Accelerators, Spectrometers, Detectors and Associated Equipment 421 (3) (1999) 531-541. doi:10.1016/S01689002(98)01208-X

[13] T. Hüyük, et al., Conceptual design of the early implementation of the neutron detector array (neda) with agata, European Physical Journal A 52 (3). doi:10.1140/epja/i2016-16055-8.

[14] E. Pollacco, et al., Must2: A new generation array for direct reaction studies, European Physical Journal A 25 (SUPPL. 1) (2005) 287-288. doi:10.1140/epjad/i2005-06-162-5.

[15] M. Labiche, et al., Tiara: A large solid angle silicon array for direct reaction studies with radioactive beams, Nuclear Instruments and Methods in Physics Research, Section A: Accelerators, Spectrometers, Detectors and Associated Equipment 614 (3) (2010) 439-448. doi:10.1016/j.nima.2010.01.009.

[16] D. Beaumel, The gaspard project, Nuclear Instruments and Methods in Physics Research, Section B: Beam Interactions with Materials and Atoms 317 (PART B) (2013) 661-663. doi:10.1016/j.nimb.2013.05.047.

[17] S. Capra, D. Mengoni, R. J. Aliaga, A. Gadea, A. Pullia, Experimental performance of the $\mathrm{I}^{2} \mathrm{C}$ integrated multichannel chargesensitive preamplifier of trace, 2015 IEEE Nuclear Science Symposium and Medical Imaging Conference (NSS/MIC) (7581814). doi:10.1109/NSSMIC.2015.7581814.

[18] S. Capra, R. J. Aliaga, D. Mengoni, P. R. John, A. Gadea, V. Herrero, A. Pullia, Evaluation of the spectroscopic performance of the integrated multi-channel charge-sensitive preamplifier of trace with a silicon detector prototype, 2016 IEEE Nuclear Science Symposium, Medical Imaging Conference and Room-Temperature Semiconductor Detector Workshop (NSS/MIC/RTSD) (8069657). doi:10.1109/NSSMIC.2016.8069657.

[19] The fbk homepage, https://www.fbk.eu/en/.

[20] N. Cieplicka-Orynczak, et al., Towards the lowest-energy limit for light ions identification with silicon pixel-type detectors, Eur. Phys. J. A, 54 (2018) 209. doi:10.1140/epja/i2018-12644-9.

[21] F. Zocca, A. Pullia, D. Bazzacco, G. Pascovici, A time-over-threshold technique for wide dynamic range gamma-ray spectroscopy with the 
agata detector, IEEE Transactions on Nuclear Science 56 (4) (2009) 2384-2391. doi:10.1109/TNS.2009.2023905.

[22] J. Gómez-Galán, R. López-Ahumada, T. Sánchez-Rodríguez, M. Sánchez-Raya, R. Jiménez, I. Martel, High speed low power fee for silicon detectors in nuclear physics applications, Nuclear Instruments and Methods in Physics Research, Section A: Accelerators, Spectrometers, Detectors and Associated Equipment 714 (2013) 155-162. doi:10.1016/j.nima.2013.03.002

[23] W. Gao, S. Li, Y. Duan, Z. Li, X. Li, Y. Hu, Sensroc11: A low-noise analog front-end readout circuit in $0.18 \mathrm{~m}$ cmos technology for czt/si-pin detectors, 2018. doi:10.1109/NSSMIC.2017.8532620. 